\title{
Retrospective estimation of exposure to benzene in a leukaemia case-control study of petroleum marketing and distribution workers in the United Kingdom
}

\author{
Stephen J Lewis, Gary M Bell, Neil Cordingley, Eileen D Pearlman, Lesley Rushton
}

\begin{abstract}
Objective-To provide quantitative estimates of exposure to benzene for cases and controls in an epidemiology study to investigate the risk of leukaemia in petroleum distribution workers.
\end{abstract}

Methods-Work histories were obtained for cases and controls together with detailed information on the distribution sites. For each job in the work history, an estimate of exposure (parts per million (ppm)) was obtained by multiplying a measure derived from exposure data by modifying factors to reflect the differences between the conditions that existed at the time of measurement and those at the time of interest. The modifying factors used related to job activity, the number of road tankers loaded, the benzene content of the gasoline, the mixture of products handled, temperature, and loading technology. Cumulative exposures for each case and control were obtained by multiplying the exposure estimates for each job by the duration of time in the respective jobs, and summing these over all jobs in the work history. Peak exposure and exposure through dermal contact were quantitatively classified for each job. Results-Measured exposures were obtained for 30 job categories, and ranged from 0.003 to $8.20 \mathrm{ppm}$. $40 \%$ of work histories were assigned background exposures, with a further $34 \%$ assigned the exposure estimate for a driver carrying out top submerged loading of motor fuel into road tankers. Cumulative exposures ranged from $<1$ to $>200$ ppm-years, although $81 \%$ were $<5$ ppm-years. Comparison of the exposure estimates for selected jobs with data from sources not used in the study showed similar results.

Conclusion-The estimates of exposure to benzene in this study provide a sound basis for the epidemiological analyses.

(Occup Environ Med 1997;54:167-175)

Keywords: benzene, retrospective exposure estimation, leukaemia, petroleum distribution industry

This paper describes the methodology for providing quantitative estimates of exposure to benzene for a retrospective nested case-control epidemiological study of leukaemia and other haematopoietic cancers in four companies in the petroleum marketing and distribution industry in the United Kingdom. The results of analyses of the leukaemia case-control study are reported in a separate paper. ${ }^{1}$

\section{History of the petroleum industry}

There were a wide variety of tasks carried out in the marketing and distribution industries varying from administration to activities which entailed regular exposure to gasoline, and as a consequence to benzene.

The period covered by the study was 1909-92 during which many operational and technological changes occurred. For example, horse drawn vehicles were replaced by road tanker vehicles which, over time, increased in size and delivered petroleum products to much wider areas. After the second world war, there was a rationalisation of terminals and the network of many small terminals was replaced by fewer larger terminals. Also, the technology used to load bulk delivery vehicles changed and, as a consequence, exposures of people engaged in loading vehicles was reduced. Most fuels were imported before 1950 when refineries were built in the United Kingdom so that it could become self sufficient in petroleum products. Refinery technology improved from straight distillation to processes which altered the composition of the hydrocarbon mixtures and produced more valuable components. These changes led to different proportions of benzene being present in gasolines. Gasolines were only a part of the total fuels handled at terminals and tended to be in higher proportions in the larger terminals. At most small terminals, $\leqslant 10 \%$ of the products handled were gasolines.

\section{Routes of exposure}

Exposure to benzene can occur in three different ways, through inhalation, skin contact, and ingestion. Inhalation is the most important route of entry into the body in the petroleum distribution industry, and ingestion the least. Published reports have generally concentrated on measurements of exposure through inhalation, with only brief references made to skin contact. In vivo experiments on human volunteers found that only $0.05 \%$ of a dose of $0.0026 \mathrm{mg} / \mathrm{cm}^{2}$ of benzene applied to the forearm skin was absorbed. ${ }^{2}$ In vitro experiments with human skin showed that the total amount absorbed seemed to increase linearly with dose and duration of exposure, and that the percentage of the applied dose absorbed at each concentration was constant at about $0 \cdot 2 \%{ }^{2}$ 


\section{Measurement techniques}

Early references ${ }^{34}$ on exposure to gasoline reported short term exposures, although some long term measurements were given in later reports. ${ }^{56}$ In general, measurements ${ }^{5-10}$ were made by adsorption of the hydrocarbon vapour on to various adsorbents then desorption and separation of the different components by gas chromatography. CONCAWE ${ }^{11-13}$ has periodically summarised data on exposure to benzene gathered from its member companies within Europe. In general, interest has focused on short term activities. The data have been collected for tasks which were considered at the time to represent the highest exposures expected-for example, the loading of gasoline into road tankers.

\section{Method}

As high quality personal exposure measurements involving full shift time weighted average (TWA) measurements have only been available since the late 1970 s, it was necessary to develop a method of estimating exposures for the early periods. Several approaches have been used to estimate exposures retrospectively and some ${ }^{14-16}$ have used multiplicative models. These estimate historical exposure by multiplying contemporary exposure results by exposure modifiers to take into account past scenarios and can be used when there are few actual measured data available. When enough actual data exist, statistical models may be more appropriately applied. The deterministic model described by $\mathrm{Yu}$ et $a l^{17}$ uses exposure modifying variables and accommodates step changes in exposure due to changes in process design or work schedules. Such effects are rep- resented by coefficients calculated from exposure data measured before and after the change under consideration, but this model requires more data than were available for this present study.

The method adopted for this study was based on a multiplicative model and extends the method used for deriving exposures for a study of Canadian petroleum distribution workers. ${ }^{18}$ The retrospective estimate of workplace exposure (WE) for each job in a work history for all study members was obtained by adjusting the base estimate (BE), a measure derived from exposure data for each job title and modifying factors $\left(\mathrm{K}_{\mathrm{i}}\right)$. These reflected the differences between the conditions that existed at the time of measurement and those at the time of interest.

This can be expressed as:

$$
\mathrm{WE}=\mathrm{BE} \times \mathrm{K}_{1} \times \mathrm{K}_{2} \times \ldots
$$

Figure 1 gives a diagrammatic representation of the workplace estimation process. The collection of information on work histories and terminals is described in a separate paper. ${ }^{1}$ The exposure estimation was carried out without knowledge of whether each study member was a case - that is, had leukaemia - or was a control.

\section{DERIVATION OF BASE ESTIMATES}

Over 200 occupational hygiene reports and articles, relating to exposure to benzene vapour in the petroleum distribution industry, were collected from the scientific literature, participating companies, and sources associated with industry. The survey reports were reviewed to establish whether the work was undertaken competently and valid sampling
Figure 1 Workplace estimation process.

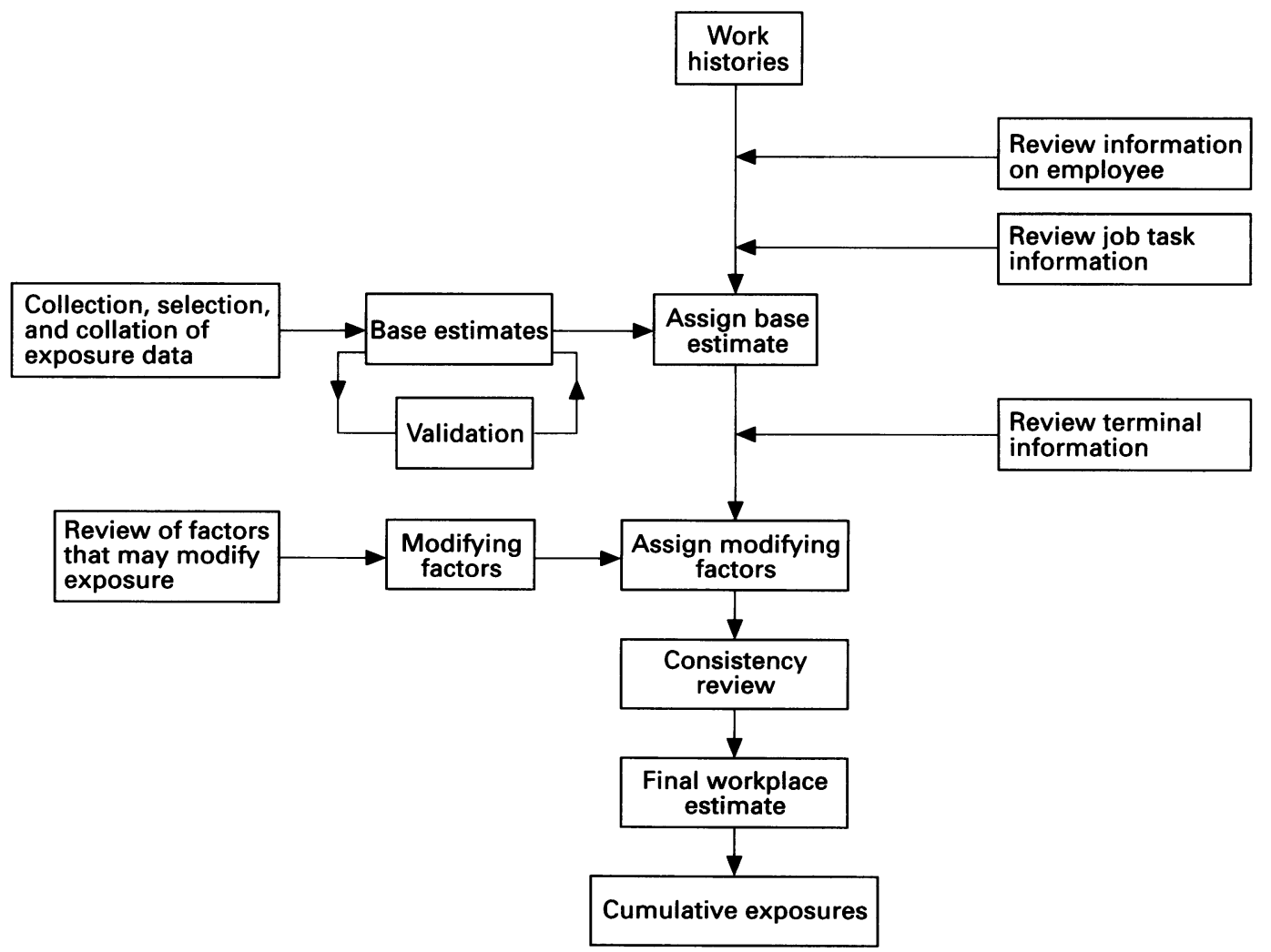


and analytical techniques were used. All exposure data were scrutinised to establish whether the modifying factors were sufficiently suitable. Insufficient characterisation of the data in many of the studies led to less than $20 \%$ of the reports collected being used for derivation of $\mathrm{BE}$.

The BEs are based on personal exposure measurements, in which samples have been collected from the breathing zone of workers. The results arising from a series of measurements can vary as a consequence of several factors including variations in the workplace, the task, the products handled, and the environment. ${ }^{19}$ Occupational hygiene data tend to fit a $\log$ normal distribution, characterised by a geometric mean (GM) and geometric SD (GSD). The arithmetic mean and GSD of the distribution (as estimated by the sample mean and sample GSD) are generally accepted as the appropriate measures of long term exposure and its variation. ${ }^{20-22}$

Benzene exposure data were taken from the occupational hygiene reports and, when necessary, standardised to $3 \%$ benzene content in the petroleum, the handling of $100 \%$ petroleum, and a temperature of $10^{\circ} \mathrm{C}$. This enabled data from different sources to be combined. These data were tested for log normality and an estimate of the long term arithmetic mean was calculated ${ }^{2324}$ and used as the value of the BE. When there were too few data points for appropriate statistical analysis, the arithmetic mean of the actual data was used. All job title BEs were 8-hour TWA concentrations, expressed in parts per million (ppm).

There were two important job titles, namely "top submerged loading drivers" and "terminal operators", for which sufficient well characterised full shift exposure measurements were unavailable and therefore a different approach was used.

TOP SUBMERGED LOADING DRIVER

The general job activities undertaken by such drivers included loading petroleum products into a road tanker at the distribution terminal, driving to retail sites, unloading the products into underground tanks, and returning to the distribution terminal. This was repeated several times a day. There were few data available on exposure to benzene during the driving and unloading activities, so additional monitoring was carried out for this study. The arithmetic mean of exposure for top submerged loading was derived from measurements standardised to a loading time of 30 minutes. It was assumed for the $\mathrm{BE}$ that three loading and unloading activities occurred each day. The 8- hour TWA BE was calculated by combining the fractional exposure associated with three loads with that for three unloading activities.

\section{TERMINAL OPERATORS}

There were few full shift benzene monitoring data available for operators. The separate tasks undertaken by terminal operators were therefore reviewed-for example, vehicle loading, tank dipping, product receipt, filter and spillage cleaning, sampling, etc. Where no or few data existed for the separate tasks additional monitoring was carried out. The existing and new data were standardised and combined according to task frequencies obtained from records and through interviews. Unlike other job titles adjustment for splash loading and the benzene concentration of the fuel was also incorporated, when appropriate, into the derived $\mathrm{BE}$.

The study was divided into five separate periods-namely, 1910-25, 1925-45, 194560, 1960-75, and 1975-92. These intervals broadly corresponded to the advent of bulk transport of fuels, the introduction of driverloaders, the approximate end of splash loading, changes in fuel use, and a reduction in manning levels, respectively. Terminals were classified into three sizes (small, medium, and large), primarily based on the number of drivers employed. This gave a total of 15 separate BEs for the job title of operator. Also, two further BEs were derived for the periods 1925-45 and 1945-60 for operators with exposure to benzene enriched fuels.

\section{DERIVATION OF THE MODIFYING FACTORS}

Factors that may have influenced exposure to benzene were extensively reviewed and were a major topic of discussion at an international workshop held at the University of Nottingham in $1993 . .^{25}$ Table 1 shows some examples of the potential modifying factors classified into four broad categories relating to the job, product, meteorology, and technology.

Other factors, such as geographical location and topography, were also considered. Several potential factors were excluded, generally because: $(a)$ the factor did not have an effect on exposure that could be shown-for example, gantry construction and site layout; (b) insufficient data were available to measure and correct for the effect of a factor-for example, wind velocity and worker position; $(c)$ the potential modifying effect occurred over relatively short periods - for example, seasonal variations.

Six modifying factors were used in the final estimation process, five of which were used in the Canadian study. ${ }^{18}$

Table 1 Examples of modifying factors considered for use in the study

\begin{tabular}{|c|c|c|c|}
\hline$f o b$ & Product & Meteorology & Technology \\
\hline $\begin{array}{l}\text { Loading activities } \\
\text { Work practices } \\
\text { Worker position } \\
\text { Frequency of activities } \\
\text { Duration of activities } \\
\text { Splitting of compartments } \\
\quad \text { during delivery }\end{array}$ & $\begin{array}{l}\text { Volume throughput } \\
\text { Benzene content of gasoline } \\
\text { Source of supply } \\
\text { Product temperature } \\
\text { Vapour pressure } \\
\text { Seasonal variation in products } \\
\text { Other products }\end{array}$ & $\begin{array}{l}\text { Air temperature } \\
\text { Wind speed } \\
\text { Wind direction } \\
\text { Seasonal changes }\end{array}$ & $\begin{array}{l}\text { Loading rate } \\
\text { Vehicle size } \\
\text { Route of supply } \\
\text { Nature of loading } \\
\text { Number of cargo compartments } \\
\text { Type of cargo measurements } \\
\text { Gantry construction } \\
\text { Site layout }\end{array}$ \\
\hline
\end{tabular}


Fob activity

A job task dictionary was compiled from various sources, including company magazines, interviews, and union records. For certain job titles, for which no measured data were available, we used the BE for the job title that most closely matched the job tasks, modified to take account of any differences.

For example: (a) Supervisors were assigned an operator BE, but assumed to have a mix of hands on and administrative duties. The degree of hands on involvement was dependent on the size of the terminal site; for larger sites it was assumed to be $10 \%$ and for smaller sites $20 \%$. (b) Drivers who drove road tankers containing black oils (fuels that did not contain benzene, such as heating fuels and bitumen) and were located at sites that handled both white oils (motor and aviation gasoline) and black oils were assumed to drive white oil vehicles one day in 20 (about two weeks a year). The $\mathrm{BE}$ for a driver handling motor gasoline was assigned and modified by a factor of 0.05 to take account of this potential exposure.

\section{Number of loads}

Loading activities were considered to be batch processes. A linear relation was used with the modifying factor being the ratio of the actual number of loads handled to the number of the loads associated with the BE. Terminal information indicated that the average numbers of loads was three and this was used as the default value. If the terminal data stated that two loads were handled a day then the appropriate modifying factor was applied-that is, $2 \div 3(0 \cdot 67)$.

\section{Percentage of benzene in the gasoline}

The percentage of benzene in gasoline varied according to its source (from which refinery in the United Kingdom or from abroad) and the year of production.

The amount of benzene vapour above a liquid gasoline can be predicted from Raoult's Law:

$$
\mathrm{VP}_{\text {benzene }}=\mathrm{PP}_{\text {benzene }} / \mathrm{MF}_{\text {benzene }}
$$

where $\mathrm{VP}_{\text {benzene }}=$ vapour pressure of pure benzene at a given temperature; $M F_{\text {benzene }}=$ the mole fraction of benzene in the liquid fuel; $\mathbf{P P}_{\text {benzene }}=$ the equilibrium partial pressure of benzene in the vapour phase above the liquid fuel.

As the mole fraction of benzene is directly proportional to the percentage of benzene, the correction factor was calculated as the ratio of the actual percentage of benzene for the gasoline under consideration divided by the standardised percentage of benzene associated with the BE (3\%). For example, gasoline with $5 \%$ benzene would have a modifying factor of $5 \div 3$, equal to $1 \cdot 67$.

Limited published retrospective data on the benzene contents of fuels were available before 1980. An exercise was carried out to estimate the average benzene content of the fuels for each of the important sources (United Kingdom refineries and imports) for each year of the period 1920-80. This involved the construction of a simple computer aided model to simulate refinery operations. The average benzene content was obtained by processing information on crude oil types, unit refinery configurations, concentrations of lead added, and required petroleum specifications and volumes.

Each terminal was assigned a refinery source(s) for different periods according to the terminal information, company information, or geographical region or location. The mean benzene content for the period associated with each job of the work history was then calculated.

\section{Mixture of products}

This factor has not been applied as such in previous studies.

There were over 300 terminals in the study and the relative quantities of petroleum handled as a function of total product varied with the different sizes of terminals. Figure 2 shows the percentage of petroleum in the total of petroleum products and except for a short period during the second world war never exceeded $50 \%$.

Small terminals tended to handle smaller proportions of petroleum, than the large terminals away from urban areas. Terminal information indicated that $<10 \%$ of the products handled at small terminals was petroleum.

The BEs were standardised to $100 \%$ petroleum handled and therefore if lower proportions were handled a modifying factor was applied. A linear relation was assumed for adjustment purposes so that a modifying factor of $0 \cdot 1(10 \div 100)$ was applied to a terminal handling only $10 \%$ petroleum. When product mix information was not directly available from site information, the modifying factor for product mix was applied according to the size of the site, the period, and the types of products handled (table 2), derived from sites where data were available.

Application of this modifying factor always has the effect of reducing exposures as the BEs are assumed to relate to handling $100 \%$ petroleum.

\section{Temperature}

The amount of vapour present above liquid gasoline is dependent on a combination of the

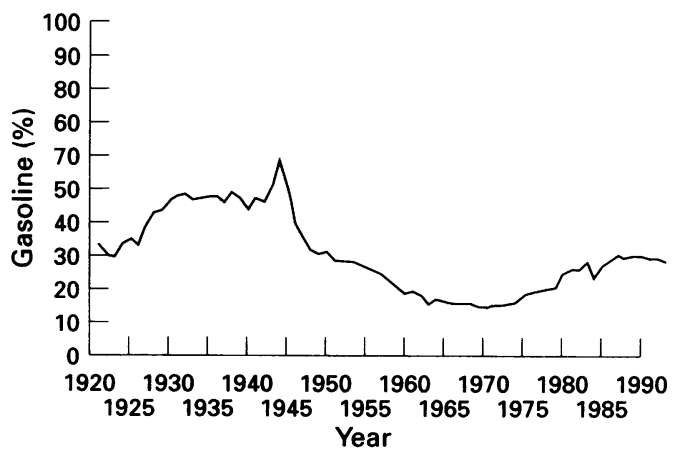

Figure 2 Percentage of gasoline in total petroleum products. 
Table 2 Product mix modifying factors for terminals without information

\begin{tabular}{llll}
\hline Terminal size & Date & Black oil & Product mix \\
\hline Small & Before 1960 & Yes & $0 \cdot 05$ \\
Small & Before 1960 & No & $0 \cdot 10$ \\
Small & After 1960 & Yes & $0 \cdot 20$ \\
Small & After 1960 & No & $0 \cdot 33$ \\
Large or medium & After 1960 & Yes & $0 \cdot 33$ \\
Large or medium & After 1960 & No & $0 \cdot 60$ \\
Large or medium & After 1960 & Yes and no & $0 \cdot 60$ \\
\hline
\end{tabular}

product and air temperatures. During the loading operation, the actual vapour temperature varies between the product temperature (from the storage tank) and the vapour temperature of the returning vehicle and residual liquid temperature. During unloading, the vapour temperature is influenced by a combination of the cargo temperature and the vapour temperature of the storage vessel. The exposure monitoring reports that recorded both air and product temperatures showed that the mean difference between these temperatures was about $1^{\circ} \mathrm{C}$. The mean annual air temperature was therefore used as a surrogate for product vapour temperature. Records of air temperature were obtained from the United Kingdom Meteorological Office. ${ }^{26}$

Vapour pressure and temperature are related by the following equation:

$$
\mathrm{P}_{\mathrm{t}}=10^{(6.893 \quad(1203.8 /(\mathrm{T}+219.91)))}
$$

where $P_{t}=$ vapour pressure; and $T=$ temperature $\left({ }^{\circ} \mathrm{C}\right)$.

The modifying factor used was the ratio of the vapour pressure at the temperature of interest to the vapour pressure at the reference temperature of $10^{\circ} \mathrm{C}$.

\section{Loading technology}

During the study period road tanker loading was carried out with both top splash and top submerged loading techniques (fig 3 ).

The filling of road tanker vehicles with product by top splash loading has not been undertaken for over 30 years as there is a risk of static discharge which could lead to explosions. There are very limited data available on emissions during this activity, although the American Petroleum Institute undertook a study reported in $1959 .{ }^{27}$ Based on the interpretation of these data, which related specifically to the differences between top splash and top submerged loading, a factor of three times the $\mathrm{BE}$ for top submerged loading was deemed appropriate to estimate the equivalent benzene exposure from top splash loading. This is the same value as that used in the Canadian study. ${ }^{18}$

Terminals built or renovated from the mid1950s were assumed to have been equipped with top submerged loading facilities with confirmation, when possible, through available site data. For sites where there was no information about the date of conversion to top submerged loading technology, 1960 was chosen for the purposes of this study, after examination of available terminal information.

\section{NON-EXPOSED PERIODS}

The total occupational exposure to benzene is the exposure during the handling of gasoline plus any exposure to benzene that might arise at work when not handling gasoline. Four of the six modifying factors relate to operational considerations-namely, the number of loads of petroleum handled each day, percentage of benzene, temperature, and loading technology. The other two factors - namely, job activity and product mix - are related to time. The total occupational exposure was calculated by the addition of the adjusted estimate of exposure to gasoline and the corresponding proportion of the background exposure when not exposed.

\section{PEAK EXPOSURE}

It was recognised that the nature of the exposure experienced for many of the jobs was not uniform, but intermittent, with the breathing zone concentrations varying during the working day as a function of the activities undertaken. It was further recognised that the nature of this type of occupational exposure could be important. Accordingly, a qualitative classification of 12 peaks was developed, characterising the intensity (two categories, 1-3, $>3 \mathrm{ppm}$ ), duration (two categories, 1-15, 15-60 minutes), and frequency (three categories, daily, weekly, monthly) of the peak. The peak categories were allocated to cases and controls on the basis of job title, terminal information, and work history comments, taking into account the possibility of short term events, such as loading of road vehicles or spillages. More than one peak category could therefore be assigned. For example, vehicle loading could give rise to a peak of 1-3 ppm intensity lasting about 30 minutes every day, but may also give rise to a higher peak $(>3$ ppm) on a weekly basis.
Figure 3 Diagrammatic representation of road tanker loading techniques.

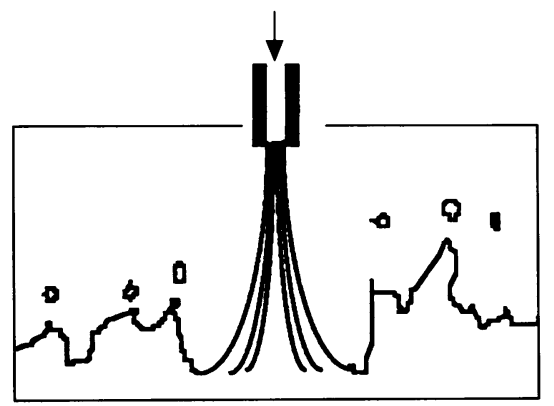

Top splash loading

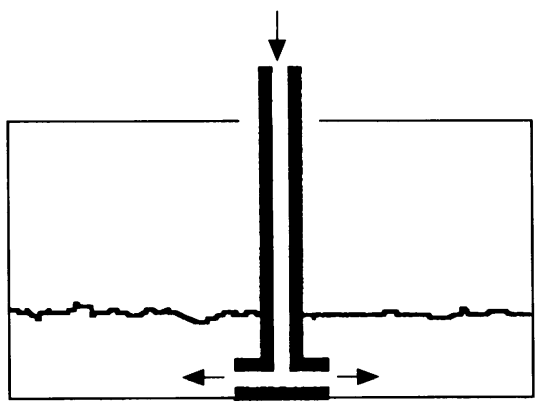

Top submerged loading 
SKIN CONTACT

Skin contact was considered but there were no reliable data for estimating quantitative exposure to benzene through the dermal route. The extent of skin contact with gasoline for each job in the work history was estimated qualitatively in one of four categories - namely, none, low, medium, or high, based on knowledge of the job tasks.

\section{Results}

The study members were drawn from a cohort of 23300 United Kingdom oil distribution workers and consisted of 91 cases of leukaemia (international classification of diseases 9th revision (ICD-9) codes 204-209) occurring before January 1993, and 364 matched controls (four per case). Altogether, 1425 independent work history lines were derived from personnel, medical, and other records and an estimate of exposure to benzene was required for each of these lines. This increased to 1616 when the period for a particular job was divided and separate estimates were made to take account of changes such as the technology or product mix, during the period.

\section{BASE ESTIMATES}

Table 3 gives the values of the BEs for each job category, the number of data points used to formulate the $\mathrm{BE}$, and the frequency of occurrence in the study.

The BEs ranged from $0.003 \mathrm{ppm}$ for those exposed to population background up to $8 \cdot 20$

Table 3 Base estimate information

\begin{tabular}{|c|c|c|c|}
\hline fob category & $\begin{array}{l}B E \\
(p p m)\end{array}$ & $\begin{array}{l}\text { Data points } \\
\text { used to } \\
\text { formulate } \\
B E(n)\end{array}$ & $\begin{array}{l}\text { Frequency of } \\
\text { occurrence } \\
\text { in the work } \\
\text { history lines } \\
\text { of the study }\end{array}$ \\
\hline Site background & 0.016 & 140 & 660 \\
\hline Small terminal, operator $1910-25$ & 0.88 & - & 1 \\
\hline Small terminal, operator $1925-45$ & 0.57 & - & 9 \\
\hline Small terminal, operator $1945-60$ & $0 \cdot 16$ & - & 40 \\
\hline Small terminal, operator $1960-75$ & $0 \cdot 18$ & - & 17 \\
\hline Small terminal, operator $1975-93$ & $0 \cdot 28$ & - & 0 \\
\hline Medium terminal, operator $1910-25$ & $2 \cdot 50$ & - & 0 \\
\hline Medium terminal, operator $1925-45$ & $2 \cdot 36$ & - & 11 \\
\hline Medium terminal, operator $1945-60$ & $2 \cdot 32$ & - & 29 \\
\hline Medium terminal, operator $1960-75$ & 0.23 & - & 31 \\
\hline Medium terminal, operator $1975-93$ & $0 \cdot 16$ & - & 11 \\
\hline Large terminal, operator $1910-25$ & 2.93 & - & 0 \\
\hline Large terminal, operator $1925-45$ & 3.49 & - & 4 \\
\hline Large terminal, operator $1945-60$ & $3 \cdot 22$ & - & 12 \\
\hline Large terminal, operator $1960-75$ & 0.68 & - & 21 \\
\hline Large terminal, operator $1975-93$ & 0.23 & - & 13 \\
\hline Benzole terminal, operator $1925-45$ & $7 \cdot 34$ & - & 6 \\
\hline Benzole terminal, operator $1945-60$ & $5 \cdot 00$ & - & 7 \\
\hline Driver without loading & $0 \cdot 13$ & 7 & 66 \\
\hline Driver top submerged loading & 0.40 & 222 & 549 \\
\hline Driver bottom loading & $0 \cdot 26$ & 8 & 2 \\
\hline Vehicle maintenance & $0 \cdot 16$ & 22 & 15 \\
\hline Population background & 0.003 & - & 26 \\
\hline Airfield operator & $0 \cdot 11$ & 62 & 34 \\
\hline Jetty operator & 1.38 & 9 & 14 \\
\hline Service station attendant & $0 \cdot 11$ & 62 & 4 \\
\hline Drum filler & $8 \cdot 20$ & 9 & 9 \\
\hline Rail car loader & $1 \cdot 27$ & 215 & 1 \\
\hline Pump and tank fitter & 0.48 & 136 & 20 \\
\hline Laboratory & $0 \cdot 19$ & 42 & 4 \\
\hline
\end{tabular}

Table 4 Lines where modifying factors were applied

\begin{tabular}{llc}
\hline Modifying factor & Times applied $n(\%)$ & Percentage increase \\
\hline Job activity & $265(16 \cdot 4)$ & $0 \cdot 6$ \\
Loads (n) & $168(10 \cdot 4)$ & $0 \cdot 1$ \\
Benzene (\%) & $784(48 \cdot 5)$ & $9 \cdot 7$ \\
Product mixture & $736(45 \cdot 5)$ & $0 \cdot 0$ \\
Temperature & $647(40 \cdot 0)$ & $11 \cdot 4$ \\
Top splash loading & $316(19 \cdot 6)$ & $19 \cdot 6$ \\
\hline
\end{tabular}

ppm for drum filling. About $40 \%$ of the work history lines were assigned background or population BEs and a further $34 \%$ were assigned the driver top submerged loading BE. Variation in the operator BEs from $0 \cdot 16$ to $7 \cdot 34$ was largely due to variation in the benzene content of the fuel, adjustment for which was incorporated in the $\mathrm{BE}$ rather than as an additional modifying factor.

\section{MODIFYING FACTORS}

On the occasions when modifying factors were not applied the factor defaulted to 1 . Table 4 gives the number and percentage of lines in which a modifying factor was applied and the number of occasions when this would have led to an increase in the $\mathrm{BE}$.

The final WE was calculated by multiplying together the BEs and all of the appropriate modifying factors. Of the resulting WEs, $6 \cdot 4 \%$ were above their respective BEs with the rest being at or below this value.

The benzene content of the motor fuels to which cases and controls were exposed were in the range $1 \%-6 \%$ by volume for normal gasolines and up to $36 \%$ in benzene enriched fuels. Before 1950, all fuel was imported and the benzene figures were $1 \%$ up to 1930 and $2 \%$ thereafter. Between 1950 and 1960 seven major refineries were built in the United Kingdom and the benzene content of the fuels varied according to a variety of factors-for example, source of crude oil, refinery configuration, and concentrations of added lead.

Table 5 gives the range of benzene contents (\% by volume), the value of the modifying factor and the frequency with which this occurred in the 1616 lines. About $40 \%$ of work history lines related to fuel with a benzene content $<3 \%$, with only $4 \%$ of lines being associated with a benzene content $\geqslant 5 \%$ by volume. There was no modification required for many lines in which either exposure was at background levels, or the lines were associated with operators in whom the adjustment for benzene content was incorporated into the $\mathrm{BE}$.

\section{WORKPLACE ESTIMATES}

Table 6 gives the frequency of workplace estimates (8-hour TWA exposure estimates (ppm)) for selected job categories, together with the mean (SD, range). As mentioned in the method, the arithmetic mean has been generally accepted as an appropriate measure of long term exposure. It can be seen that the mean exposure estimates for all these jobs was well below $1 \mathrm{ppm}$. The ranges of the WEs (table 6) were fairly small except for drivers and operators. For example, the range for drivers who carried out top loading was 0.02 to $14 \cdot 40$. The maximum value of 14.40 was derived by modifying the BE of 0.4 for this job (table 3 ) for a benzene content of $36 \%$ (modifying factor = 12 , see method for percentage of benzene in gasoline), and for splash loading (modifying factor $=3$, see method for loading technology).

Cumulative exposures were calculated for each case and control by summing the workplace estimates over all the lines of the work history. Figure 4 shows the frequency of 
Table 5 Range and frequency of occurrence of the modifying values for benzene content

\begin{tabular}{llc}
\hline Benzene content (\%) & Modifying value & Frequency \\
\hline $1 \cdot 0-1 \cdot 9$ & $0 \cdot 33-0 \cdot 63$ & 245 \\
$2 \cdot 0-2 \cdot 9$ & $0 \cdot 67-0.97$ & 431 \\
$3 \cdot 0-3 \cdot 9$ & $1 \cdot 00-1 \cdot 30$ & 98 \\
$4 \cdot 0-4 \cdot 9$ & $1 \cdot 33-1 \cdot 63$ & 39 \\
$5 \cdot 0-9 \cdot 9$ & $1 \cdot 67-3 \cdot 30$ & 31 \\
$10 \cdot 0-14 \cdot 9$ & $3 \cdot 33-4 \cdot 97$ & 1 \\
$15 \cdot 0-19 \cdot 9$ & $5 \cdot 00-6 \cdot 63$ & 12 \\
$20 \cdot 0-29 \cdot 9$ & $6 \cdot 67-9 \cdot 67$ & 16 \\
$>30$ & $>10$ & 1 \\
\multicolumn{2}{l}{ No modification } & 742 \\
\hline
\end{tabular}

Table 6 Data associated with the workplace estimates

\begin{tabular}{|c|c|c|c|c|}
\hline \multirow[b]{2}{*}{ fob category } & \multirow[b]{2}{*}{ Observations ( $n$ ) } & \multicolumn{2}{|c|}{ Exposure (ppm) } & \multirow[b]{2}{*}{ Range } \\
\hline & & mean & $S D$ & \\
\hline Airfield operator & 34 & 0.05 & 0.02 & $0 \cdot 02-0 \cdot 11$ \\
\hline Driver, no loading & 66 & 0.03 & 0.01 & $0.02-0.07$ \\
\hline Driver, top loading & 549 & $0 \cdot 48$ & 1.43 & $0 \cdot 02-14 \cdot 40$ \\
\hline Jetty operator & 14 & $0 \cdot 37$ & 0.25 & $0.09-0.92$ \\
\hline Operator & 209 & 0.53 & $1 \cdot 19$ & $0 \cdot 02-7 \cdot 34$ \\
\hline Pump and tank fitter & 20 & $0 \cdot 46$ & 0.07 & $0 \cdot 25-0.48$ \\
\hline Vehicle maintenance & 15 & $0 \cdot 10$ & 0.04 & $0.09-0.24$ \\
\hline
\end{tabular}

occurrence of the cumulative benzene exposures (ppm-years) for all the cases and controls, (the distribution was similar for both).

Cumulative exposures ranged from $<0.1$ to $>200$ ppm-years, although $81 \%$ were $<5$ ppm-years.

\section{INFLUENCE OF APPLYING A PRODUCT MIX} MODIFYING FACTOR

Figure 5 shows the range of WEs calculated during this study together with the WEs that would have been obtained if the product mix modifying factor had not been applied.

The mean cumulative exposure for the people in the study was 4.61 ppm-years. If the product mix factor had not been applied the mean would have been 8.08 ppm-years. The graph indicates that the greatest effect from applying this factor is in the higher exposure ranges. This is due to low proportions of gasolines being handled during the earlier decades of the study, when exposures were at their highest-for example, during splash loading.

COMPARISON WITH OTHER DATA

An evaluation of the representativeness of selected BEs was carried out by comparing them with data from sources not used in this

Figure 4 Cumulative exposures of all study members.

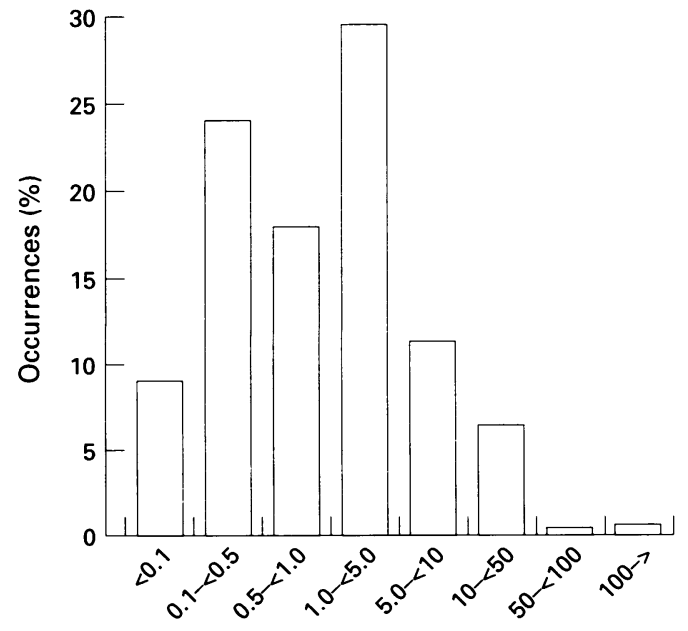

Range of cumulative exposures (ppm-y)

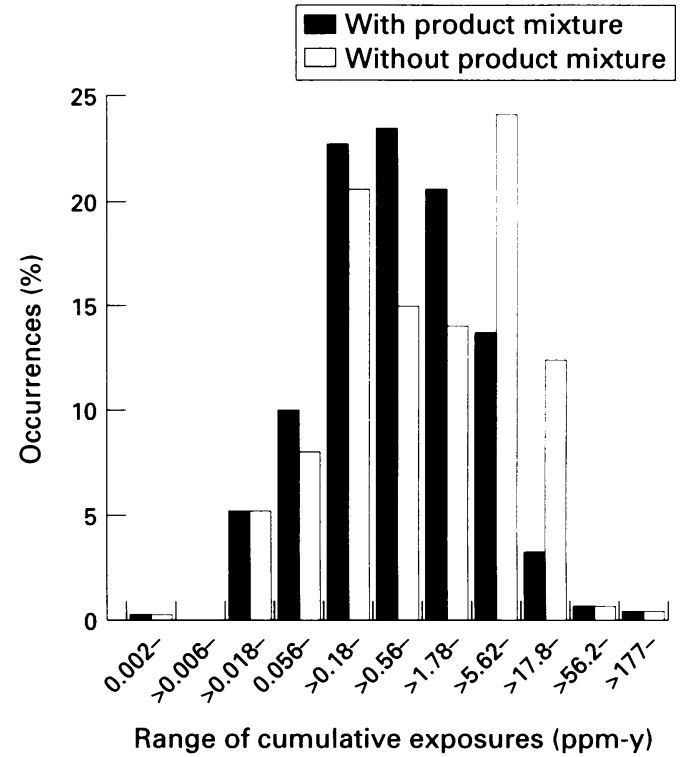

Figure 5 Range of workplace estimates with and without the application of a product mixture modifying factor.

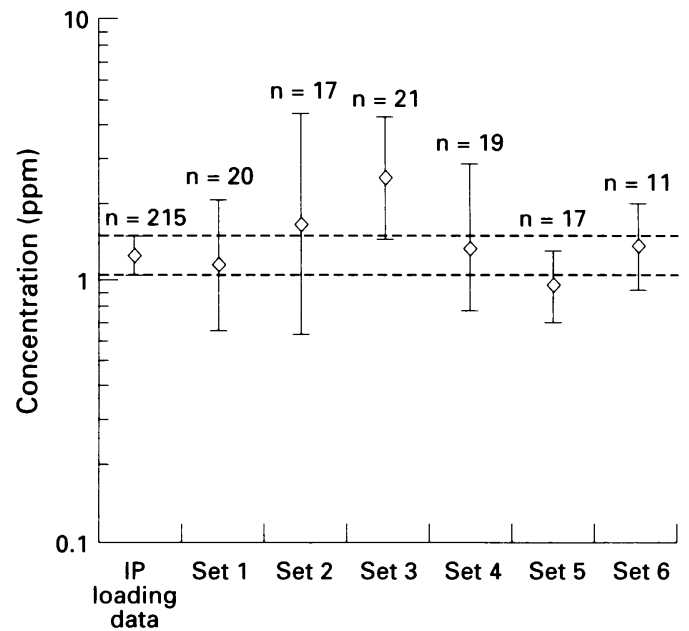

Figure 6 Comparison of mean top submerged loading base estimate value from the IP study with means (95\% CI) from data from six other United Kingdom sites.

study. Figure 6 shows a comparison of the BE for drivers using top submerged loading from this study with six other data sets obtained from United Kingdom sites. (These were not included in the derivation of the study BE because information on all the modifying factors was not available.) The $95 \%$ confidence intervals (95\% CIs) on the study data are narrow as they are derived from 215 data points. Where possible all data have been standardised, by correcting to $10^{\circ} \mathrm{C}, 3 \%$ benzene, and $100 \%$ gasoline. The graph shows that the study loading exposure data fell within the $95 \%$ CIs of all of the comparison data sets, and the study mean value fell within the $95 \%$ CIs for five out of the six comparison sets.

A monitoring exercise was undertaken in the United Kingdom in 1969 by Parkinson ${ }^{3}$ during the handling of benzene enriched fuels (10\%-33\% benzene). The WE was calculated with the algorithm used during this study. This was carried out by modifying the top submerged loading driver BE for time, tempera- 
Table 7 Comparison with the Parkinson ${ }^{3}$ data

\begin{tabular}{|c|c|c|c|c|c|}
\hline $\begin{array}{l}\text { Base } \\
\text { estimate } \\
\text { (ppm) }\end{array}$ & $\begin{array}{l}\text { Sampling } \\
\text { time } \\
\text { (min) }\end{array}$ & $\begin{array}{l}\text { Benzene in } \\
\text { gasoline } \\
(\%)\end{array}$ & $\begin{array}{l}\text { Temperature } \\
\left({ }^{\circ} \mathrm{C}\right)\end{array}$ & $\begin{array}{l}\text { Study WE } \\
\text { benzene } \\
\text { (ppm) }\end{array}$ & $\begin{array}{l}\text { Parkinson }{ }^{3} \\
\text { measured } \\
\text { benzene } \\
(p p m)\end{array}$ \\
\hline $\begin{array}{l}0 \cdot 40 \\
0 \cdot 40 \\
0 \cdot 40 \\
0 \cdot 40 \\
0 \cdot 40\end{array}$ & $\begin{array}{r}90 \\
150 \\
120 \\
60 \\
90\end{array}$ & $\begin{array}{l}33 \cdot 0 \\
33 \cdot 0 \\
33 \cdot 0 \\
20 \cdot 0 \\
10 \cdot 0\end{array}$ & $\begin{array}{l}20 \cdot 0 \\
20 \cdot 0 \\
20 \cdot 0 \\
18 \cdot 0 \\
17 \cdot 0\end{array}$ & $\begin{array}{r}12 \cdot 9 \\
7 \cdot 8 \\
9 \cdot 7 \\
10 \cdot 7 \\
3 \cdot 4\end{array}$ & $\begin{array}{l}5 \cdot 7 \\
4 \cdot 5 \\
6 \cdot 7 \\
6 \cdot 7 \\
3 \cdot 3\end{array}$ \\
\hline
\end{tabular}

ture, number of loads, and benzene content. Table 7 gives the results of this comparison and indicates that the derived values are higher than the exposures measured in 1969 by factors between one and $2 \cdot 3$.

A similar exercise was carried with data from a study of Canadian distribution workers. ${ }^{18}$ The BE for drivers carrying out top submerged loading was $0.40 \mathrm{ppm}(95 \%$ CI $0 \cdot 21-0.58$ ) for this study, compared with 0.71 ppm $(95 \%$ CI $0 \cdot 40-1 \cdot 40)$ for the Canadian study, assuming a product mix of $85 \%$.

\section{Discussion}

This study has provided quantitative estimates of exposure to benzene in petroleum distribution workers over almost 80 years.

Personal exposures measured under known conditions were modified to take account of differing conditions. Although many reports containing relevant occupational hygiene measurements were available, fewer than $20 \%$ included comprehensive information on the modifying factors. Also, personal monitoring data for people in the study were not available and representative full shift data were also limited for many activities carried out in petroleum distribution terminals. Additional information gathered during surveys could have provided a greater characterisation of the conditions. This would have led to a more comprehensive understanding of the prevailing conditions and more useful data for subsequent use and comparison. The additional time and cost involved to collect the information would have been very small compared with the overall survey period. It was necessary to undertake additional monitoring on some short term activities and combine these and other measured data to create BEs for two of the most important job titles.

Six important modifying factors which affect exposure to benzene have been taken into account, where appropriate-namely those pertaining to job activity, the number of loads, benzene content of fuel, mix of products handled, temperature, and loading technology.

Although information on work histories and the operating sites was collected in a systematic and consistent way, uncertainties inherent in these data may contribute to uncertainties in the exposure estimation. For example, certain terminal information collected during interviews was contradictory and, on occasions, discrepancies of up to 10 years were highlighted when compared with documentary evidence.
The exposure values obtained by the method used should be treated as estimates of average exposure that characterise the long term exposures experienced during each task under consideration. Limited differentiation of individual study members was possible from the interview information available, but it was not possible to allow for variation in worker practice between and within sites.

An assumption was made that in the long term, operators at a terminal would work the same proportion of time at each of the tasks associated with the job title. Although the study did not attempt to measure the effect of dermal contact or intermittent exposures, it did consider these issues in a qualitative manner.

Although it is often considered that exposures to benzene must have been greater in the past, there is no evidence of this within the United Kingdom petroleum distribution industry. This was largely due to the benzene content of the fuels being lower in the past and many of the small terminals handled relatively small proportions of fuels containing benzene. Also, petroleum distribution was not a renowned "dirty" industry. The hazards of mishandling gasoline and other inflammable materials have long been acknowledged and articles from company magazines, as early as the 1900 s, which highlighted the need to handle products carefully to avoid such hazards and the cost associated with product spillages and loss. This is also reflected in the mortality statistics of the cohort study of United Kingdom petroleum distribution workers, where no excess of deaths due to accidental fire and explosion was found. ${ }^{28}$

It was not possible to quantify the levels of exposure due to skin contact in this study. However, the proportion of benzene absorbed by the skin has been shown experimentally to be very small. ${ }^{2}$ The industrial hygiene practices and the use of protective equipment such as gloves would also have greatly reduced the potential for dermal contact.

It was possible to compare the exposure data for selected activities with published sources of information not used for the study. The results of six such comparisons showed comparability in four instances with one overestimate (factor of 2) and one underestimate (factor of between 1 and $2 \cdot 2$ ).

\section{Conclusions}

The estimates of exposure to benzene obtained in this study form a basis for an epidemiological analysis, and enable an assessment of the risk of leukaemia to be evaluated. Further work could include research into the appropriate methods for the development of ranges around the derived estimates and the measurement of intermittent exposures.

The advent of detailed health and safety legislation that requires more formal and structured health risk assessments, will lead industry to collect and retain more comprehensive data associated with workplace activities. 
The United Kingdom Institute of Petroleum (IP) funded and supported the study. We thank our technical adviser, Tom Armstrong (Exxon Biomedical Sciences) for his considerable support, advice, and practical help, and Catherine Bell and Kathryn Holliday for their assistance during the exposure estimating process. The staff at the University of Nottingham provided critical support to the exposure assessment task force, and we particularly thank Sarah Grace and Helena Romaniuk. We also thank the University of Warwick, and Russ Stevens for their assistance in providing information used during the study. The occupational hygienists who formed the core exposure estimating team were strongly supported by $\mathrm{Dr}$ Michael Claydon, Dr Michael Molyneux, and Jim Sanderson. Finally, we thank the IP Steering Group and the Scientific Advisory we thank the IP Steering Group and the Scientific Advisory
Board (particularly Dr John Steel) for their advice and guidBoard (particularly Dr John Steel) for their advice and guidance. The IP Steering Group organised the important survey undertaken by John Hayes and Bernard Patrick on estimates of
the historical benzene content of United Kingdom motor fuels.

1 Rushton L, Romaniuk HM. A case-control study to investigate the risk of leukaemia associated with exposure to benzene in petroleum marketing and distribution workers in the United Kingdom. Occup Environ Med 1997;54: 152-66.

2 Franz TJ. Percutaneous absorption of benzene. In: McFarland HN, Holdsworth CE, MacGregor JA, eds.
Advances in modern environmental toxicology. Vol VI. Advances in modern environmental toxicology. Vol VI. New Jersey: Princetown Sci Publ, 1984:61-70.

3 Parkinson GS. Benzene in motor gasoline-An investigation into possible health hazards in and around filling stations and in normal transport operations. Ann Occup Hyg 1971;14:155-7.

4 Sherwood RJ. The monitoring of benzene exposure by air sampling. Am Ind Hyg Assoc 7 1971;32:840-6.

5 McDermott HJ, Vos GA. Service station attendants' exposure to benzene and gasoline vapors. Am Ind Hyg Assoc $\mathcal{F}$ 1979;40:315-21.

6 Irving WS, Grumbles TG. Benzene exposures during gasoline loading at bulk marketing terminals. Am Ind Hyg Assoc $\mathcal{F} 1979 ; 40: 468-73$

7 Sherwood RJ, Carter FWG. The measurement of occupational exposure to benzene vapour. Ann Occup Hyg 1970; 13:125-46.

8 Sherwood RJ. Evaluation of exposure to benzene vapour during the loading of petrol. $B r \mathcal{F}$ Ind Med 1972;29:65-9.

9 National Institute for Occupational Safety and Health. Manual of analytical methods. Cincinnati: NIOSH, 1974. (HEW Publication No (NIOSH) 75-121.)

10 Anonymous. Method for monitoring exposure to gasoline vapour in air. The Hague: CONCAWE, 1986. (Report 8/86.)

11 Tims JM. Exposure to atmospheric benzene vapour associated with motor gasoline. The Hague: CONCAWE, 1981. with motor gasoline.
12 Christian F, Eyres AR. Review of European oil industry benzene exposure data. The Hague: CONCAWE, 1986. (Report no $3 / 86$.)

13 Review of European oil industry benzene exposure data (1986-92). Brussels: CONCAWE, 1994. (Report no 7/94.)

14 Dodgson J, Cherrie J, Groat S. Estimation of past exposure to respirable man-made mineral fibres in the European insulation wool industry. Ann Occup Hyg 1987;31: 567-82.

15 Schneider T, Olsen I, Jorgensen O, Lauersen B. Evaluation of exposure information. Appl Occup Environ Hyg 1991; 6:475-81.

16 Griefe AL, Hornung RW, Stayner LG, Steenland KN. Development of a model for use in estimating exposure to ethlyene oxide in a retrospective cohort mortality study. Scand f Work Environ Health 1988;14(suppl 1): 29-30.

17 Yu RL, Tan W, Mathew RM, Andjelkovich DA, Levine RJ. A deterministic mathematical model for quantitative estimation of historical exposure. Am Ind Hyg Assoc $\mathcal{F}$ 1990;51:194-201.

18 Armstrong TW, Pearlman ED, Schnatter AR, Bowes SM, Murray N, Nicholich MJ. Retrospective benzene and total hydrocarbon exposure assessment for a petroleum marketing and distribution worker epidemiology study. Am Ind Hyg Assoc F 1995; (in press).

19 Nicas MJ, Simmons BP, Spear RC. Environmental versus analytical variability in exposure measurements. Am Ind analytical variability in exp

20 Rappaport SM. Assessment of long-term exposures to toxic substances in air. Ann Occup Hyg 1991;35:61-121.

21 Rappaport SM. Selection of the measures of exposure for epidemiology studies. Appl Occup Environ Hyg 1991;6: 448-57.

22 Smith TJ. Exposure assessment for occupational epidemiology. Am F Ind Med 1987;12:249-68.

23 Armstrong BG. Confidence intervals for arithmetic means of lognormally distributed exposures. Am Ind Hyg Assoc $\mathcal{F}$ 1992;53:481-5.

24 Morris JF, Koski A, Johnson LC. Spirometric standards for healthy non-smoking adults. Am Rev Respir Dis 1971; 103:57-67.

25 Rushton L, Thar WE. Retrospective exposure assessment for benzene: issues, methods, and recommendations from an international workshop on petroleum marketing and distribution worker studies. Occup Hyg 1996;3: 295-305.

26 Metereological Office. Air mean temperatures-fanuary to December 1961-90. London: Metereological Office, 1991.

27 American Petroleum Institute. Evaporation loss from tank cars, tank trucks, and marine vessels. New York: API 1959. (API bulletin 2514.)

28 Rushton L Further follow up of mortality in a United Kingdom oil distribution centre cohort. $\mathrm{Br} \mathcal{f}$ Ind $\mathrm{Med}$ 1993;50:561-9. 